\title{
Application of a simple method for determining the equilibrium resistivity of dielectric liquids
}

\author{
Joachim Schiessling, Nils Lavesson, Lars Walfridsson
}

ABB Corporate Research, Västerås, Sweden

\begin{abstract}
For the design of the electrical insulation of an UHVDC (Ultra-High Voltage Direct Current) converter transformer the resistivity of the pressboard and mineral oil has to be known. For solid insulation the resistivity is virtually constant, however, in liquids it is dependent on, e.g., applied electric stress and time of electrification. Several methods are discussed in the standards. One way to characterize the oil is the measurement of the equilibrium resistivity, in contrast to an apparent resistivity for oil under high electric stress. Measurements using a low voltage triangular shaped voltage form allow calculation of equilibrium resistivity. The oils investigated show equilibrium resistivity in the range $5 \times 10^{11} \Omega \mathrm{m}$ to $5.96 \times 10^{12} \Omega \mathrm{m}$, which are typical values for transformer oil. There is good agreement between these results and reference measurements using dielectric response.
\end{abstract}

\section{Introduction}

Ultra-High Voltage Direct Current (UHVDC) is the method of choice to transfer power over long distance with low losses. Each UHVDC system consists of at least two converter stations to convert AC to DC power or vice versa. Key components of such a station are converter transformers, placed in between the AC grid and the DC valve hall. A converter transformer is a large power transformer that in addition to $\mathrm{AC}$ has to withstand DC electric stress. The insulation of a large power transformer consists mainly of pressboard and mineral oil. In AC power transformers the stress on the electrical insulation is determined by the permittivity of the materials, while for converter transformers the resistivity of the materials have to be taken into account. The permittivity is constant and differs between materials in the order of a factor of two. Resistivity however is more complex; it can vary by several orders of magnitude between materials and for mineral oil it is a function of electric stress, time of electrification, etc. This dependence makes a characterization of mineral oil difficult for DC applications. A number of approaches are discussed in the past ranging from measurements using very low voltages (equilibrium resistivity) to high fields (stressed oil) [1]. To get a representative result for a dielectric liquid the equilibrium resistivity should be measured, thus only small electric field should be used.
One standard method is based on dielectric response measurements [2] where a sinusoidal voltage is applied and the current response is measured. A method that typically implies long measurement time to obtain equilibrium resistivy. A simple method to measure resistivity is proposed by Gäfvert et al. [3] by application of a triangular shaped voltage, probing the equilibrium state with low fields. An application of this method is described in this text.

\section{Experimental details}

\section{The triangular method}

The measurement cell is assumed to have electrodes with an area A spaced a distance d apart, where the distance $d$ is much smaller than the distance from edge to edge of the electrode. The current measured with the triangular method is described by the following equation [3]

$$
I(t)=\frac{A}{\rho d} U(t)+\frac{\varepsilon_{r} \varepsilon_{0} A}{d} \frac{d U}{d t}
$$

where $\rho$ denotes the resistivity and $\varepsilon_{\mathrm{r}}$ and $\varepsilon_{0}$ are the relative permittivity and the permittivity of vacuum. As seen in the equation above, the current has a resistive part, proportional to applied voltage, and a capacitive part, proportional to the change in voltage. With knowledge of the ratio $\mathrm{A} / \mathrm{d}$ the resistivity $\rho$ is calculated. The part proportional to the change in voltage is the displacement current resulting in a jump in the measured current.

\section{Experimental set up}

For the present measurements two test cells from IRLAB are used, see Fig. 1. Both cells have a cylindrical geometry, CL-1 has a ratio of electrode area to electrode distance of $\mathrm{A} / \mathrm{d}=2.56 \mathrm{~m}, \mathrm{CL}-2$ has a ratio of $\mathrm{A} / \mathrm{d}=2.64 \mathrm{~m}$. A special feature of CL-2 is that it can be used to measure resistivity of flowing oil, however, this option has not been used for the present measurements. Before filling, the cells are cleaned with isopropanol and acetone. Different batches of mineral oil (labeled A, B and C) are investigated. 


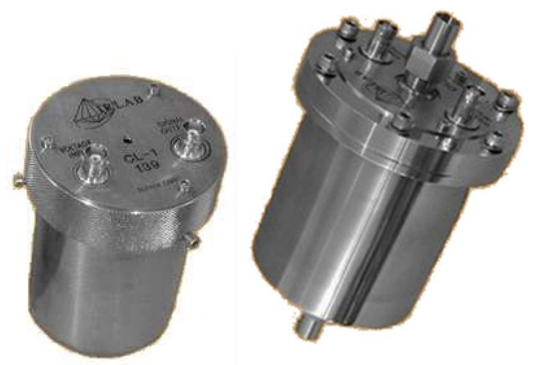

Fig. 1 - Test cells used. LHS CL-1 and RHS CL-2. For CL-2 has connections for hoses to measure flowing oil.

The test cell is connected with BNC cables to a function generator and an electrometer. A Yokogawa FG120 function generator is used to apply a triangular voltage. A Keithley 6517B electrometer is used to measure current. Both units are connected via GPIB to a computer that runs a LabView program to control the signal generator and read current from the Keithley. The current data is analyzed and the equilibrium resistivity of the oil is calculated. The experimental setup is shown schematically in Fig. 2.

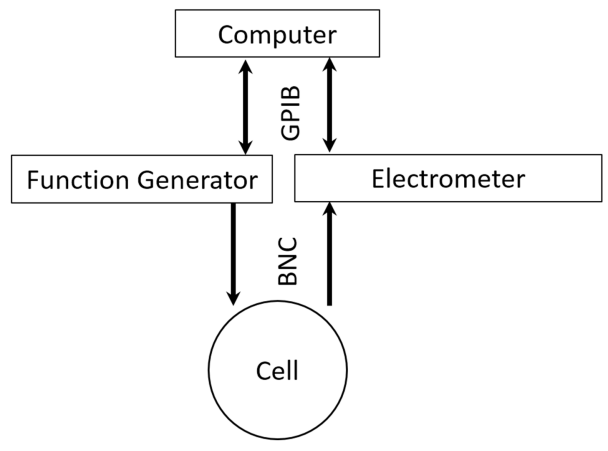

Fig. 2 - Schematic of experimental set-up for triangular wave method.

\section{Reference Measurements}

In order to benchmark the results, reference measurements are carried out using the respective test cell connected to a commercial IDA200 system. This system is designed for dielectric response measurements, from which the equilibrium resistivity can be obtained. The wave form used is a sinusoidal shape with amplitude of $2 \mathrm{~V}$ at a frequency of $0.1 \mathrm{~Hz}$ to $0.01 \mathrm{~Hz}$.

\section{Results}

A typical current measurement is shown in Fig. 3 and the linear part of the current is easily identified. The applied voltage is $12 \mathrm{~V}$ and the increase in current over one half period is in the order of a $6 \mathrm{pA}$. In this set-up, the equipment is adequately shielded and the noise level is in the order of $0.5 \mathrm{pA}$.

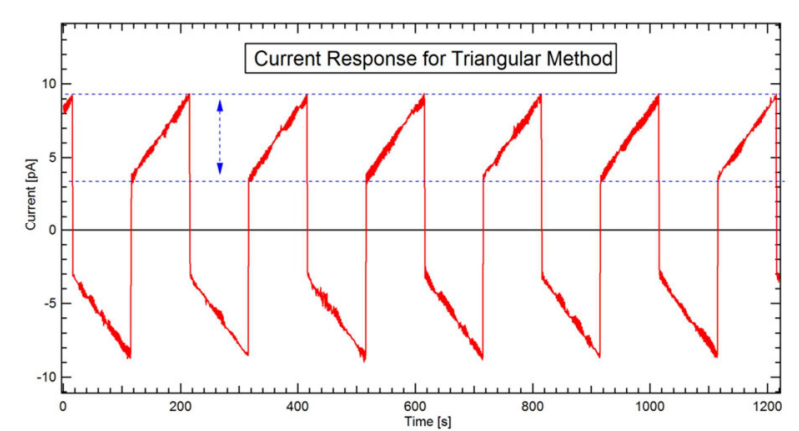

Fig. 3- Typical current data for oil A. Applied $12 \mathrm{~V}$ amplitude and $0.005 \mathrm{~Hz}$ for oil with resistivity of $5.9 \times 10^{12} \Omega \mathrm{m}$. Dashed lines and arrow indicate the rise in current that is used for the estimation of resistivity. Similar analysis can be done for the negative values.

Calculated resistivity in terms of applied frequency and voltage is shown in Fig. 4. Amplitudes range from $0.5 \mathrm{~V}$ to $12 \mathrm{~V}$ and frequency from $0.005 \mathrm{~Hz}$ to $0.08 \mathrm{~Hz}$. Data from dielectric response measurements are shown for comparison. There is a clear dependence on frequency, but no voltage dependence is seen in the results. For lower frequencies, there is good agreement for data from the triangular method compared to IDA data. At approx. $0.02 \mathrm{~Hz}$ both data set start to diverge with IDA data showing reduced resistivity, whereas data from triangular method increases in resistivity.

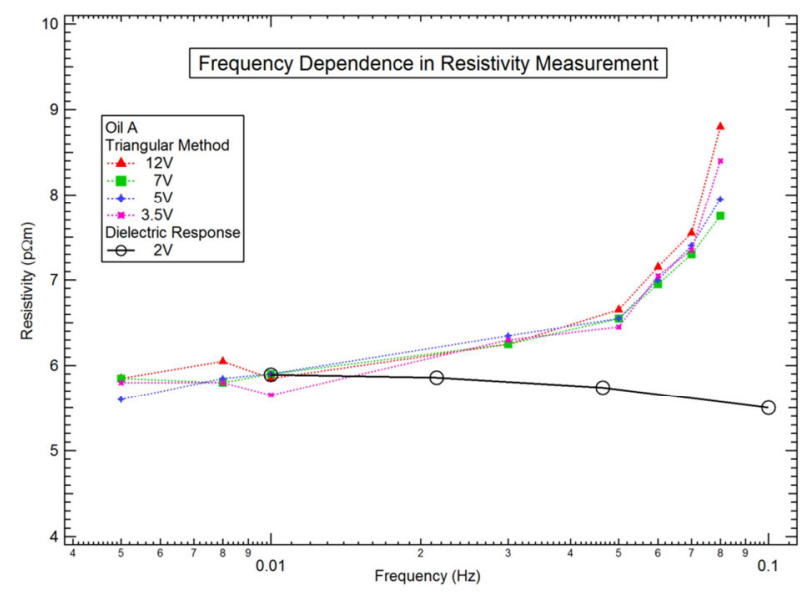

Fig. 4 - Frequency dependence of resistivity estimated from triangular method and dielectric response in range $0.05 \mathrm{~Hz}$ to $0.1 \mathrm{~Hz}$.

Experimental results for different ranges of resistivity are shown in Fig. 5. The cells are filled with different batches of mineral oil. Oil A and B are measured in CL1 , oil $\mathrm{C}$ in cell CL-2. Oil B is measured several times, since a larger fluctuation in the data is observed. Dielectric response measurements are shown for comparison. Regarding the frequency behavior, the same general trend is seen for all oils. At lower frequencies, the data is flat and there is good agreement between the triangular method and dielectric response. 


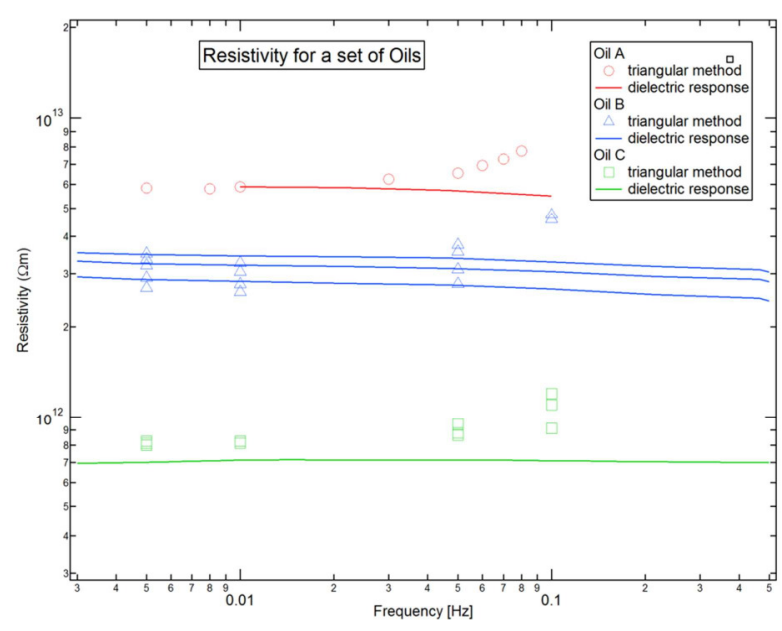

Fig. 5- Resistivity data for a number of oils and a number of frequencies compared to data from IDA measurements. Oil B repeated measurements are shown.

\section{Discussions}

The measurements shown above are carried out for triangular waves with an amplitude from $0.5 \mathrm{~V}$ to $12 \mathrm{~V}$ and frequencies ranging from $0.005 \mathrm{~Hz}$ to $0.1 \mathrm{~Hz}$. The general observation is that the calculated resistive is flat for lower frequency and at approx. $0.05 \mathrm{~Hz}$ the resistivity increases with frequency. This is an artefact and can attributed to the contribution from the displacement current; it is simply more difficult to separate the contribution of the small conduction current from the much larger displacement current.

The opposite behavior is observed for the results from the dielectric response measurement, where the resistivity decreases with increasing frequency. This is caused by increased dielectric losses in oil. The dependence on frequency in the range investigated is not as strong as seen for the triangular method, however, approximately the same threshold frequency is observed. Comparing the results from both methods, there is good agreement for frequencies below $0.05 \mathrm{~Hz}$.

Beside the frequency, the noise level is the other limitation for the triangular method. The noise level in the current data is not a hinder for the measurement and suggest that even more resistive oil can be measured without problems, e.g. oils with one order of magnitude larger resistivity. A resistivity of $5 \times 10^{13} \Omega \mathrm{m}$ is rather extreme for industrial application and reflects a very high resistive oil.

In the measurements above, no strong influence of the amplitude of the applied triangular voltage on the results is observed. In principle, it should be low enough to keep the oils close to equilibrium condition and it should be chosen so that the resulting current is significant larger than the noise level. The frequency of the applied triangular wave should be as low as possible in order to minimize influence of displacement current, however, too long measurement times have drawbacks experimentally.
In dielectric response measurements, typically a frequency sweep is carried out and resistivity values are chosen in a range where the results show no dependence on frequency. The procedure is time consuming.

The advantage of the discussed triangular wave method is the option to continuously monitor the measured current. By doing this, both frequency and voltage of the triangular wave can be adjusted during a measurement by evaluating the shape of the current, e.g. judging the contribution of the displacement current and noise level.

\section{Conclusions}

In general, there is good agreement between the triangular method and the reference dielectric response measurement for all oils investigated. The limit of the method is for high resistive oil, since currents are small. As a rule of thumb, a recommendation is to use a frequency of $0.001 \mathrm{~Hz}$ and a voltage in the range of 1$10 \mathrm{~V}$. The advantage is the simplicity of the method and the possibility to optimize the measurement frequency due to online monitoring of the current shape.

\section{References}

[1] Cigré Technical Brochure TB 646 January 2016 "HVDC Transformer Insulation: Oil conductivity"

[2] IEC 60247 "Insulating liquids - Measurement of relative permittivity, dielectric dissipation factor $(\tan \delta)$ and DC resistivity"

[3] U. Gäfvert, H. Kols, and J. Marinko. NORDIS 1990 "Simple method for determining the electric conductivity of dielectric liquids" 